\title{
ON THE THROUGHPUT OF OPPORTUNISTIC BEAMFORMING WITH IMPERFECT CSI
}

\author{
Ali Vakili, Amir F. Dana, and Babak Hassibi \\ California Institute of Technology \\ Department of Electrical Engineering \\ Pasadena, CA 91125, USA
}

\begin{abstract}
The throughput of a multiple-antenna broadcast channel highly depends on the channel state information (CSI) at the transmitter side. Due to the time variant nature of wireless channels, having perfect knowledge of the underlying links appears to be a questionable assumption, especially when the number of users and/or antennas increases.

For a broadcast channel with $M$ transmit antennas and $n$ users, each provided with one receive antenna, it is shown that opportunistic beamforming can achieve the same scaling as that of dirty paper coding for large $n$. Although the dirty paper coding scheme needs perfect CSI from all users, opportunistic beamforming needs very little feedback. In this paper we investigate the performance of opportunistic beamforming when the CSI is not perfect; i.e., the channel estimation is erroneous. We will show that in order to maximize the throughput, the transmitter needs to back off the rate than what is suggested by the estimated channel coefficient. We obtain the optimal back off and show that with this modified opportunistic scheme, the same multiuser gain will be achievable; in other words, the throughput scales like $M \log \log n$ even with imperfect limited CSI.
\end{abstract}

Index Terms - Broadcast channel, MIMO systems, channel state information(CSI), estimation error, multiuser diversity.

\section{INTRODUCTION}

The main framework for studying the down-link of cellular systems is the MIMO Gaussian broadcast channel. Therefore a great deal of research has been conducted into the study of the capacity of MIMO Gaussian broadcast channels. It is shown [1] that the entire capacity region is achievable using the dirty paper coding (DPC) [2].

However, the computational complexity and full channel state information (CSI) requirement by make DPC infeasible in practice. Therefore other schemes are proposed which are suboptimal, but require little feedback. Moreover,

This work was supported by NSF grant CCR-0133818, by Caltech's Lee Center for Advanced Networking and by a grant from the David and Lucille Packard Foundation. these schemes are not computationally intensive like DPC. One such scheme is opportunistic beamforming [3] in which the transmitter selects a number of random beams and the users feed back their highest SINR among the SINR's they receive on different beams. Based on there estimates, the transmitter assigns each beam to the user with the highest SINR on that beam. However, in the analysis of the performance of this scheme the CSI is assumed to be perfect (i.e., no error in the channel estimation process), which may not be the case especially when the number of users and/or antennas increases.

As of the related work, in [4] the authors study the effect of channel estimation error on the capacity region of MIMO Gaussian broadcast channels under DPC scheme. The throughput of opportunistic scheme in a single-antenna broadcast channel has been studied in [5]. The effect of imperfect CSI on the capacity of point-to-point SISO and MIMO channels is considered in [6], [7], and [8]. The robustness of the capacity achieving scheme in a Gaussian flat-fading channel is studied in [9]. As for the broadcast channel, [10] has considered the effect of non-ideal feedback only in the transmitter due to Doppler effect.

In this paper we will look at the robustness of opportunistic beamforming against the erroneous channel estimation. In fact in the scheme that we will consider the users feed back their highest channel coefficient instead of their highest SINR. We will look at the throughput scaling law in the regime of large number of users. When perfect CSI is available, it has been shown that opportunistic beamforming follows the same scaling law as that of DPC. We will show that in order to maximize the throughput, the transmitter has to assume some value for the actual SINR of the underlying link based on the estimated channel coefficient. We will show that the throughput scales like $M \log \log n$ which is exactly the same scaling obtained by DPC.

The remainder of this paper is organized as follows. Section 2 describes the formulation of the problem along with the proposed transmission scheme. In Section 3, we obtain the optimal assumed SINR. The scaling of the throughput will be obtained for the regime of large $n$ in Section 4. Finally, Section 5 concludes the paper. 


\section{PROBLEM FORMULATION}

\section{Channel Model}

In this paper we consider a multiple-antenna broadcast channel with $M$ transmit antennas and $n$ users, each provided with $N=1$ receive antenna. Users are assumed to have uncorrelated Gaussian block-fading channels with a coherence time equal to $T$ channel uses. Therefore each user's channel can be described by a $M \times 1$ vector, $H_{i}$, which is constant during the coherence time and has independent $\mathcal{C N}(0,1)$ entries. We denote the transmitted signal at $t$-th channel use by a $M \times 1$ vector, namely $S(t), t=1, \ldots, T$. Thus the signal received by the $i$-th user at $t$-th channel use can be written as

$$
y_{i}(t)=H_{i}^{*} S(t)+w_{i}(t)
$$

where $w_{i}(t)$ denotes the additive Gaussian noise of zero mean and variance $\rho$ (the network is assumed to be homogenous). Moreover, the total transmit power is M; i.e., $E\left[S^{*}(t) S(t)\right]=$ $M$.

\section{Random Beamforming}

We assume that for each channel coherence interval the transmitter chooses $M$ orthonormal $M \times 1$ random beams denoted by $\phi_{m}, m=1, \ldots, M$, from an isotropic distribution. At the $t$-th channel use $M$ symbols are transmitted by choosing $S(t)=\sum_{m=1}^{M} \phi_{m} s_{m}(t)$. Therefore $y_{i}(t)$ can be rewritten as

$$
y_{i}(t)=\sum_{m=1}^{M} x_{i, m} s_{m}(t)+w_{i}(t)
$$

in which $x_{i, m}=H_{i}^{*} \phi_{m}$. All $x_{i, m}$ 's have $\mathcal{C N}(0,1)$ distribution and are constant during the coherence interval. Moreover, it is straightforward to check that $E\left[x_{i, m}^{*} x_{i^{\prime}, m^{\prime}}\right]=\delta_{i, m} \delta_{i^{\prime}, m^{\prime}}$.

We assume that at the beginning of each coherence interval the users estimate their channels. The estimate of $x_{i, m}$ will be denoted by $\hat{x}_{i, m}$. We will use $\tilde{x}_{i, m}$ to denote the error in the estimation of $x_{i, m}$. Thus,

$$
x_{i, m}=\hat{x}_{i, m}+\tilde{x}_{i, m} .
$$

By assuming a MMSE estimator, $\hat{x}_{i, m}$ and $\tilde{x}_{i, m}$ will be independent and will have $\mathcal{C N}(0,1-\beta)$ and $\mathcal{C N}(0, \beta)$ distributions, respectively. Each user sends back its highest estimated channel coefficient, i.e. $\max _{m}\left|\hat{x}_{i, m}\right|^{2}$ along with its index. Throughout the paper we will use $m_{i}$ to denote this index. The transmitter assigns the $m$-th beam to the user with the best corresponding estimated channel, i.e. $\max _{i}\left|\hat{x}_{i, m}\right|^{2}$. We will denote this user by $i_{m}$. It can be shown that [3] the probability that one user is the best user on two different beams is negligible as the number of users, $n$, grows. Furthermore, when a user is selected to be served on the $m$-th beam, the data intended for that user is encoded into $s_{m}(t)$ using a Gaussian capacity-achieving codebook.

\section{Average Throughput}

If the $m_{i}$-th beam is assigned to the $i$-th user, we can rewrite $y_{i}(t)$ as,

$$
y_{i}(t)=x_{i, m_{i}} s_{m_{i}}(t)+\sum_{m \neq m_{i}} x_{i, m} s_{m}(t)+w_{i}(t),
$$

in which the second term is the interference caused by the other beams not intended for that user. Since $s_{m}$ 's are chosen from a Gaussian codebook and the data streams for different users are independent, this interference term affects the signal like an additive Gaussian noise. It is shown that [11] in this scenario the receiver does not need to know the channel, and any rate less than the channel capacity will be achievable (see also [12] and the discussion in [5]). The capacity can be expressed as $\log \left(1+S I N R_{i, m_{i}}\right)$, in which $S I N R_{i, m_{i}}$ denotes the signal-to-noise-plus-interference-ratio at the $m_{i}$-th beam at the $i$-th receiver; i.e.,

$$
S I N R_{i, m_{i}}=\frac{\left|x_{i, m_{i}}\right|^{2}}{1 / \rho+\sum_{m^{\prime} \neq m_{i}}\left|x_{i, m^{\prime}}\right|^{2}} .
$$

While serving a user with the estimated channel strength of $\left|\hat{x}_{i, m}\right|^{2}$, the transmitter needs to choose a transmission rate which is less than $\log \left(1+S I N R_{i, m_{i}}\right)$. We assume that any transmission at a rate higher than the capacity fails. In other words, the instantaneous of the $i$-th user, $R_{i}$ will be,

$$
R_{i}= \begin{cases}\log \left(1+\lambda_{i_{m}}\right) & \lambda_{i_{m}} \leq S I N R_{i, m_{i}} \\ 0 & \lambda_{i_{m}}>S_{N} N R_{i, m_{i}}\end{cases}
$$

Throughout the paper we will refer to $\lambda_{i_{m}}$ as the assumed SINR of the $i$-th user which is a function of $\hat{x}_{i_{m}, m}$. The average throughput of this scheme can be expressed as,

$$
R_{a v}=\sum_{m=1}^{M} E_{\hat{x}_{i_{m}, m}} R_{i_{m}}\left(\hat{x}_{i_{m}, m}\right)
$$

in which

$$
\begin{aligned}
& R_{i_{m}}\left(\hat{x}_{i_{m}, m}\right)= \\
& \quad \max _{\lambda_{i_{m}}} \operatorname{Pr}\left(\lambda_{i_{m}} \leq S I N R_{i_{m}, m} \mid \hat{x}_{i_{m}, m}\right) \log \left(1+\lambda_{i_{m}}\right) .(9)
\end{aligned}
$$

In the following section we will solve the above maximization problem in order to find the optimum assumed SINR.

\section{THE OPTIMUM ASSUMED SINR}

Due to the symmetry of the problem, $R_{i_{m}}$ does not depend on $m$ and we can focus on a particular beam, say the first one. Thus,

$$
R_{a v}=M E_{\hat{x}_{i_{1}, 1}} R_{i_{1}}\left(\hat{x}_{i_{1}, 1}\right) .
$$

First, we have to calculate,

$$
P_{1}=\operatorname{Pr}\left(\lambda_{i_{1}} \leq \frac{\left|x_{i_{1}, 1}\right|^{2}}{1 / \rho+\sum_{m^{\prime} \neq 1}\left|x_{i_{1}, m^{\prime}}\right|^{2}} \mid \hat{x}_{i_{1}, 1}\right),
$$


in which $y=\sum_{m^{\prime} \neq 1}\left|x_{i_{1}, m^{\prime}}\right|^{2}$ represents the sum of the squares of $2(M-2)$ Gaussian independent random variables. Thus $y$ will have a chi-square distribution with a CDF which can expressed as,

$$
F_{Y}(y)=1-e^{-y} \sum_{j=1}^{M-2} \frac{1}{j !} y^{j}, \quad y \geq 0
$$

Moreover, by fixing the value of $\hat{x}_{i_{1}, 1}$, one can check that $z=\left|x_{i_{1}, 1}\right|^{2}$ has a Rician distribution of the form,

$$
f_{Z}(z)=\frac{1}{\beta} e^{-\frac{z+\left|\hat{x}_{i_{1}, 1}\right|^{2}}{\beta}} I_{0}\left(\frac{2\left|\hat{x}_{i_{1}, 1}\right| \sqrt{z}}{\beta}\right)
$$

Therefore $P_{1}$ can be obtained from,

$$
P_{1}=\int u\left(\frac{z}{\lambda_{i_{1}}}-\frac{1}{\rho}-y\right) f_{Y}(y) f_{Z}(z) d y d z,
$$

where $u($.$) denotes the unit step function. The above integral$ can be rewritten as,

$$
P_{1}=\int F_{Y}\left(\frac{z}{\lambda_{i_{1}}}-\frac{1}{\rho}\right) f_{Z}(z) d z
$$

We will consider the regime of large number of users. Since $\left|\hat{x}_{i_{1}, 1}\right|^{2}$ is the maximum among $n$ independent random variables with exponential distribution, it will scale like $\log n$. Thus we can assume that $\left|\hat{x}_{i_{1}, 1}\right|^{2} \gg 1$. Furthermore, we can say intuitively that $\lambda_{i_{1}}$ has to be large. Therefore we can approximate the Bessel function in the above integral by its asymptotic value, i.e.

$$
I_{0}(x) \rightarrow \frac{e^{x}}{\sqrt{2 \pi x}}
$$

By plugging (16) into (13) and using saddle point integration we can approximate (15) by,

$P_{1}=1-e^{-\left(\frac{\left|\hat{x}_{i_{1}, 1}\right|^{2}}{\lambda_{i_{1}}}-\frac{1}{\rho}\right)} \sum_{j=1}^{M-2} \frac{1}{j !}\left(\frac{\left|\hat{x}_{i_{1}, 1}\right|^{2}}{\lambda_{i_{1}}}-\frac{1}{\rho}\right)^{j}+O\left(\frac{1}{(\log n)^{3}}\right)$

In order to find the best assumed SINR in the regime of large number of users, one has to solve the following maximization problem,

$$
\max _{\lambda_{i_{1}}} e^{-\left(\frac{\left|\hat{x}_{i_{1}, 1}\right|^{2}}{\lambda_{i_{1}}}-\frac{1}{\rho}\right)} \sum_{j=1}^{M-2} \frac{1}{j !}\left(\frac{\left|\hat{x}_{i_{1}, 1}\right|^{2}}{\lambda_{i_{1}}}-\frac{1}{\rho}\right)^{j} \log \left(1+\lambda_{i_{1}}\right)
$$

Although analytically solving the above maximization problem is intractable, in the regime of large number of users it can be simplified further in order to find an approximate solution. The following theorem gives a reasonable approximation for the optimum assumed SINR, $\lambda_{i_{1}}^{*}$.
Theorem 1. Consider a broadcast channel with $n$ users and a transmitter equipped with $M>1$ antennas which employs opportunistic beamforming based on the estimated highest channel coefficient. Assume that at a channel coherence interval the $i_{m}$-th user has the highest estimated channel coefficient for the $m$-th beam, given by $\left|\hat{x}_{i_{m}, m}\right|^{2}$. If the transmitter serves this user at a rate equal to $\log \left(1+\lambda_{i_{m}}\right)$, where

$$
\lambda_{i_{m}}^{*}=\frac{\left|\hat{x}_{i_{m}, m}\right|^{2}}{\frac{2}{\rho}+(M-1) \log (M-1)+\log \frac{\rho \log \left|\hat{x}_{i_{m}, m}\right|^{2}}{(M-2) !}}
$$

the instantaneous rate (averaged on the coherence interval) will be,

$$
R_{i_{m}}=\log \left(\left|\hat{x}_{i_{m}, m}\right|^{2}\right)\left(1+O\left(\frac{\log \log \log \left|\hat{x}_{i_{m}, m}\right|^{2}}{\log \left|\hat{x}_{i_{m}, m}\right|^{2}}\right)\right) .
$$

Proof: look at [13].

We have to remark that although $P_{1}$ and $\lambda_{i_{1}}^{*}$ can be calculated numerically with little effort, the simulation results suggest that (17) and (19) give relatively good approximations for these two quantities, respectively.

\section{THE SCALING OF SUM RATE CAPACITY IN THE REGIME OF LARGE NUMBER OF USERS}

In order to find the throughput of this scheduling as expressed in (10), we need the distribution of $\left|\hat{x}_{i_{1}, 1}\right|^{2}$. The pdf of $\hat{x}_{i, m}$ can be written as,

$$
f_{\hat{X}}\left(\hat{x}_{i, m}\right)=\frac{1}{1-\beta} e^{-\frac{\left|\hat{x}_{i, m}\right|^{2}}{1-\beta}}
$$

As mentioned earlier, all $\hat{x}_{i, m}$ 's are independent for different $i$ or $m$ 's and are identically distributed. Therefore the pdf of $\max _{i}\left|\hat{x}_{i_{1}, 1}\right|^{2}$ can be found using the fact that,

$f_{X_{\max }}\left(x_{\max }=\max _{i=1, \ldots, n} x_{i}\right)=f_{X}\left(x_{\max }\right)\left(1-F_{X}\left(x_{\max }\right)\right)^{n-1}$,

where $x_{i}$ 's are i.i.d. random variables. We can state the following theorem:

Theorem 2. Consider a broadcast channel with the setting of Theorem 1. The transmitter employs opportunistic beamforming and modifies the transmission rate as described in Theorem 1. The throughput of the system can be found to be,

$$
R_{a v}=M \log \log n+O(\log \log \log \log n) .
$$

Proof: As we have mentioned before, in the regime of large $n$, the distribution of $\left|\hat{x}_{i_{1}, 1}\right|^{2}$ becomes more and more concentrated around $\log n$, such that the main contribution to $R_{a v}$ comes from the interval of $\log n \pm \log \log n$. For details look at [13] • 


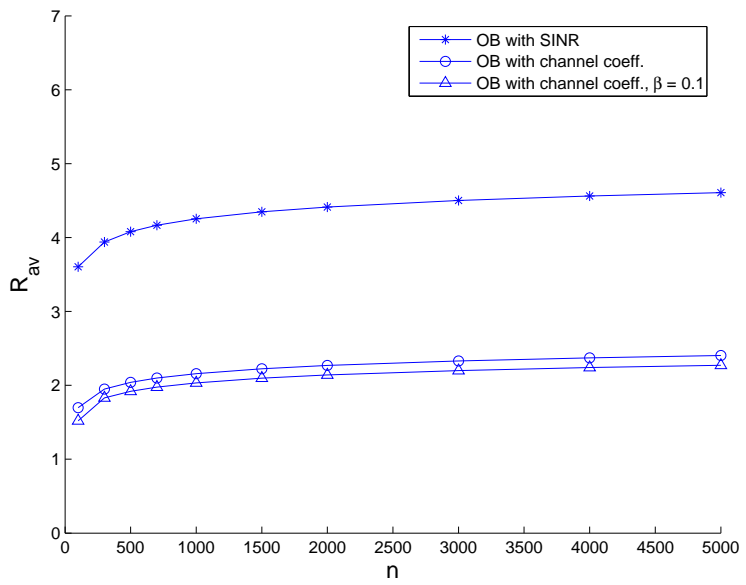

Fig. 1. Throughput of opportunistic beamforming

As the above theorem states, the multiuser diversity gain, $M \log \log n$, is achievable in a multiple-antenna broadcast channel even with imperfect knowledge of the highest channel coefficients of all users. This gives the same scaling law as that of the dirty paper coding and random beamforming based on the precise values of SINR's. However, the second highest order term of the asymptotic behavior of the throughput does not match that of the opportunistic beamforming with perfect CSI. As mentioned earlier, in the scheme we have considered in this paper, for the sake of simplicity of the analysis we have assumed that the users feed back their highest channel coefficient instead of their highest SINR.

In Figure 1, the achieved rate for three cases are plotted as a function of $n$. The first case is the opportunistic beamforming with the feedback of the SINR values. The second scenario is the opportunistic beamforming with no estimation error when the transmitter only knows the channel coefficients. In the third scenario we have a channel estimation error with the error variance of $\beta=0.1$. The plots are generated for $M=2$ antennas and for $\rho=1$.

\section{CONCLUSION}

We considered a multiple-antenna Gaussian broadcast channel. We looked at the throughput achieved by opportunistic beamforming in the presence of channel estimation error. In the scheme proposed here, the users feed back their highest channel coefficient along with the index of the beam for which they have the best channel. The transmitter assigns each beam to the user with the highest estimated channel coefficient for that beam. For the regime of large number of users, we showed that the scaling law of the throughput of such scheme is precisely the same as the scaling law of the throughput in the absence of channel estimation error.

\section{REFERENCES}

[1] H. Weingarten, Y. Steinberg, and S. Shamai (Shitz), "The capacity region of the Gaussian MIMO broadcast channel," in Proc. of Conf. on Information Sciences and Systems, March, 2004.

[2] M. Costa, "Writing on dirty paper," IEEE Trans. Inform. Theory, vol. 29, no. 3, pp. 439-441, May 1983.

[3] M. Sharif and B. Hassibi, "On the capacity of MIMO broadcast channels with partial side information," IEEE Trans. Inform. Theory, vol. 51, no. 2, pp. 506-522, Feb. 2005.

[4] A. F. Dana, M. Sharif, and B. Hassibi, "On the capacity region of multi-antenna Gaussian broadcast channels with estimation error," in Proc. of ISIT, July, 2006.

[5] A. Vakili and B. Hassibi, "On the throughput of broadcast channels with imperfect CSI," in Proc. of SPAWC, July, 2006.

[6] M. Medard, "The effect upon channel capacity in wireless communications of perfect and imperfect knowledge of the channel," IEEE Trans. on Information Theory, vol. 46, no. 3, pp. 933-946, May 2000.

[7] B. Hassibi and B. M. Hochwald, "How much training is needed in a multiple-antenna wireless link?," IEEE Trans. on Information Theory, vol. 48, no. 5, pp. 11181134, May 2003.

[8] T. Yoo and A. Goldsmith, "Capacity and optimal power allocation for fading MIMO channels with channel estimation error," Submitted to IEEE Trans. on Information Theory, August 2002.

[9] A. Lapidoth and S. Shamai, "Fading channels: How perfect need "perfect side information" be?," IEEE Trans. on Information Theory, vol. 49, no. 10, pp. 951-964, April 2002.

[10] M. Kobayashi, G. Caire, and D. Gesbert, "Antenna diversity vs. multiuser diversity: Quantifying the tradeoffs," in Proc. ISITA, October, 2004.

[11] N. Merhav, G. Kaplan, A. Lapidoth, and S. Shamai, "On information rates for mismatched decoders," IEEE Trans. on Information Theory, vol. 40, no. 6, pp. 19531967, November 1994.

[12] C. E. Shannon, "Probability of error for optimal codes in a Gaussian channel," Bell Systems Technology Journal, vol. 38, pp. 611-656, May 1959.

[13] A. Vakili, A. F. Dana, and B. Hassibi, "On the throughput of opportunistic beamforming with imperfect CSI," in preparation. 